\title{
Capicua is involved in Dorsal-mediated repression of zerknüllt expression in Drosophila embryo
}

\author{
Dong-Hyeon Shin E Joung-Woo Hong * \\ Graduate School of East-West Medical Science, Kyung Hee University, Yongin 446-702, Korea
}

\begin{abstract}
The maternal transcription factor Dorsal (DI) functions as both an activator and a repressor in a context-dependent manner to control dorsal-ventral patterning in the Drosophila embryo. Previous studies have suggested that $\mathrm{Dl}$ is an intrinsic activator and its repressive activity requires additional corepressors that bind corepressor-binding sites near Dl-binding sites. However, the molecular identities of the corepressors have yet to be identified. Here, we present evidence that Capicua (Cic) is involved in Dl-mediated repression in the zerknüllt (zen) ventral repression element (VRE). Computational and genetic analyses indicate that a DNA-binding consensus sequence of Cic is highly analogous with previously identified corepressorbinding sequences and that DI failed to repress zen expression in lateral regions of cic mutant embryos. Furthermore, electrophoretic mobility shift assay (EMSA) shows that Cic directly interacts with several corepressor-binding sites in the zen VRE. These results suggest that Cic may function as a corepressor by binding the VRE. [BMB Reports 2014; 47(9): 518-523]
\end{abstract}

\section{INTRODUCTION}

The maternal effect gene, dorsal $(d l)$, encodes a Rel-homology domain (RHD) transcription factor, which is related to mammalian NFKB (1). Like NFKB, DI is also regulated the level of nuclear transport, leading to the formation of a DI nuclear gradient, with peak levels of protein present in ventral regions and progressively lower levels in lateral and dorsal regions in early Drosophila embryos (2). The nuclear gradient of DI activates sequential expression of patterning genes along the dorsal-ventral (DV) axis of the embryo, thereby establishing three different germ layers, the presumptive mesoderm, the neurogenic ectoderm, and the dorsal ectoderm (3).

*Corresponding author. Tel: +82-31-201-3853; Fax: +82-31-2048119; E-mail: jwhong46@khu.ac.kr

http://dx.doi.org/10.5483/BMBRep.2014.47.9.122

Received 5 June 2014, Revised 24 June 2014, Accepted 27 June 2014

Keywords: Capicua, Corepressor, Dorsal-mediated repression, Drosophila embryo, VRE, Zerknüllt
In contrast to its role as an activator, $\mathrm{DI}$ is also able to act as a repressor that restricts the expression of decapentaplegic (dpp) and zerknüllt (zen) to dorsal regions, where they are responsible for the differentiation of the dorsal epidermis and the amnioserosa, respectively $(4,5)$. The cis-regulatory elements responsible for Dl-mediated repression have been studied in detail at the zen locus. DI represses the ventral expression of zen through a distal regulatory region, called the ventral repression element (VRE), which is located about $1.2 \mathrm{~kb}$ upstream from the zen transcription start site (Fig. 1A) (6). Several lines of evidence suggest that repression by $\mathrm{Dl}$ requires additional factors, or "corepressors." First, DI binding sites found in the zen VRE and the twi enhancer are functionally interchangeable (6). Namely, a DI site in zen VRE mediates activation when it is multimerized and located upstream of a heterologous promoter. Conversely, when a Dl site from a twi enhancer is placed within the zen VRE, it mediates transcriptional repression. These findings indicate that the sequence of the DI binding site alone does not seem to determine whether Dl functions as an activator or a repressor. Second, in addition to these three critical Dl sites, the zen VRE contains five AT-rich sites for an unknown factor, three of which lie adjacent to the DI sites (Fig. 1B) (7). The repression activity of the VRE requires strict spacing between at least one of the AT-rich sites (AT2) and the linked DI site (8), suggesting that there are cooperative DNA-binding interactions between the proteins binding the two sites. Third, mutations in the AT-rich sites adjacent to the DI sites in the VRE lead to derepression of zen expression in the lateral regions (7). Taken altogether, it is conceivable that $\mathrm{Dl}$ inherently acts as a transcriptional activator and, in order to function as a repressor, it must interact with corepressors bound to neighboring sites. Although there have been efforts to identify the corepressors in the context of the Drosophila embryo, the proteins that function as the DI binding partner remain unknown.

Here, we present evidence that a product of the maternal effect gene, capicua (cic), is involved in Dl-mediated repression in the zen VRE. Computational and genetic analyses showed that sequences of the AT-rich sites found in VRE are included in a consensus DNA-binding sequence for $\mathrm{Cic}$, produced by a high-throughput bacterial-one hybrid $(\mathrm{B} 1 \mathrm{H})$ system (9), and that zen expression was ventrally extended in cic mutant embryos. Furthermore, an electrophoretic mobility shift assay 
(EMSA) showed that recombinant Cic is able to specifically bind the AT-rich sites. These results suggest that cooperative site occupancy by $\mathrm{Dl}$ and Cic converts the intrinsic activator $\mathrm{Dl}$ into a repressor.

\section{RESULTS}

The AT-rich sequences in the zen VRE may be a subset of the Cic DNA binding consensus sequence

The approximately $600 \mathrm{bp}$ VRE sequence contains three Dl-binding, four Zelda (Zld)-binding, and five unknown factor-binding sites (Fig. 1B and S1 in Supplementary Material) (7). The Zld-binding sites are occupied by a zinc-finger maternal transcription activator, Zld, which allows the VRE to medi-
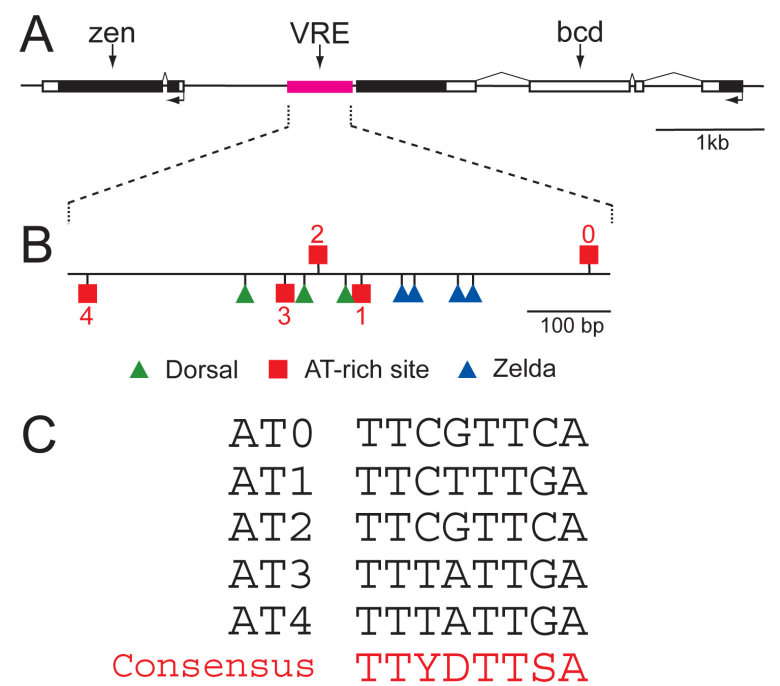

D

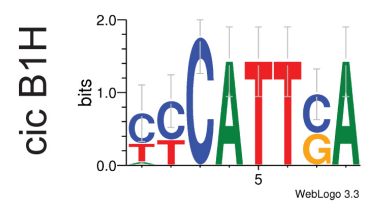

Fig. 1. The AT-rich sequences in the zen VRE may be a subset of the Cic DNA-binding consensus sequence. (A) $\mathrm{A} \sim 7-\mathrm{kb}$ genomic region encompassing the zerknüllt (zen) locus: The ventral repression element (VRE) is located between -1.6 and -1 kb upstream of the transcription start site of zen. The 5 ' end of the VRE abuts the 3' end of the last exon of bicoid (bcd). (B) The $\sim 600$ bp VRE contains three Dorsal (Dl, green triangle), five unknown factors (AT-rich site, red square), and four Zelda (Zld, blue triangle) binding sites (Fig. S1). (C) Aligned AT-rich sequences found in the VRE are shown and the consensus sequence is TTYDTTSA. IUPAC nucleic acid codes for $Y, D$, and $S$ represent $\mathrm{C}$ or $\mathrm{T}, \mathrm{A}, \mathrm{T}$ or $\mathrm{G}$, and $\mathrm{C}$ or $\mathrm{G}$, respectively. (D) DNA-binding sequences for Cic generated by the bacterial-1-hybrid $(\mathrm{B} 1 \mathrm{H})$ system are graphically represented as sequence logos (22). The WebLogo 3.3 algorithm (http://weblogo.berkeley.edu/) was used to build the sequence logo with the position frequency matrix (PFM) of Cic binding sequences (Fig. S3). ate ubiquitous expression of zen in syncytial blastoderm embryos (10). The unknown factor-binding sites have been called AT-rich sites because those sequences are closely related to each other and contain an AT-rich core with the consensus sequence 5'-TTYDTTSA-3' (Fig. 1C). Previous P-element-mediated transformation and site-directed mutagenesis analyses suggest that three (AT1, AT2 and AT3) of the five AT-rich sites in the VRE are essential for Dl-mediated repression $(7,8)$.

To identify any protein factors that bind those AT-rich sites in

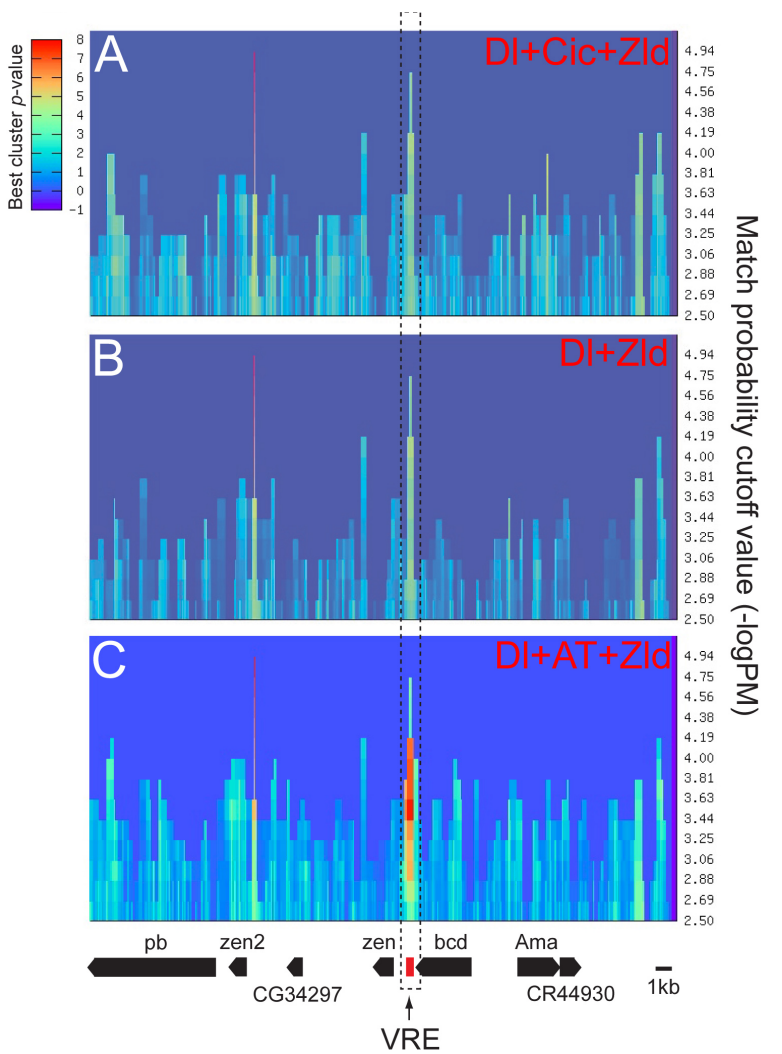

Fig. 2. The ClusterDraw analysis of a $\sim 40-k b$ genomic region encompassing a zen locus. ClusterDraw analyses with PFMs of Dl, Cic and Zld shows two statistically significant match probability values, the second highest of which corresponds to the VRE (A). A pattern obtained by ClusterDraw analysis with PFMs of DI and Zld (B) is comparable with that in panel A. The same analysis except with PFMs of Dl, AT sites (Fig. S2), and Zld indicates that the VRE region contains the highest cluster $\mathrm{P}$-value as well as the second highest match probability value, compared with the above two analyses (C). These results suggest that PFM of Cic produced by the $\mathrm{B} 1 \mathrm{H}$ system functionally diverged from that of AT-rich sites at least in the computational analyses, presumably because of one nucleotide mismatch between PFMs of Cic and AT-rich sites. The second highest match probability values produced by the ClusterDraw analyses and their corresponding genomic regions containing the VRE are indicated by the dotted box. Gene organization over the analyzed genomic region was depicted in the bottom of panel $\mathrm{C}$. The VRE located between zen and bcd is represented as a red box. See Supplementary Materials for more details. 
the zen VRE, the library of Drosophila transcription factor binding motifs, recently constructed by the bacterial 1-hybrid $(\mathrm{B} 1 \mathrm{H})$ system (9), was searched using a position-frequency matrix (PFM) of the AT-rich sites (Fig. S2). The search results revealed that the $\mathrm{B} 1 \mathrm{H}$ database contains only one transcription factor binding motif whose consensus sequence, 5'-YYCATTSA-3', is highly analogous to that of AT-rich sites (Fig. 1D, compare with 1C). The DNA motif retrieved from the $\mathrm{B} 1 \mathrm{H}$ database is occupied by a high mobility group (HMG)-box transcription factor, Capicua (Cic). The cic is a maternal effect gene whose expression pattern is ubiquitous throughout the embryo, at least until the onset of nuclear cleavage cycle 14 (11), which is considerably superimposed in space and time with the observed Dl-mediated repression pattern. Thus, it is conceivable that the cic protein may be able to bind the AT-rich sites in the VRE.

To test whether the consensus sequence of Cic can functionally represent the AT-rich sequences found in the VRE, we calculated the statistical significance of cluster formation between $\mathrm{Dl}, \mathrm{Cic}$, and Zld in a zen locus using the ClusterDraw algorithm (Fig. 2), which is an $r$-scan-based program that has been developed to identify binding motifs and binding clusters of a specified combination of transcription factors (12). When given the PFMs of transcription factors of interest and the maximum cluster size, ClusterDraw calculates cluster significance from the sum of consecutive distances between all binding motif matches present in a cluster and determines the statistical significance of every possible cluster in a given DNA sequence (100 kb maximum). Thus, we hypothesized that if Cic functions by binding the AT-rich sites in the VRE, the PFM of
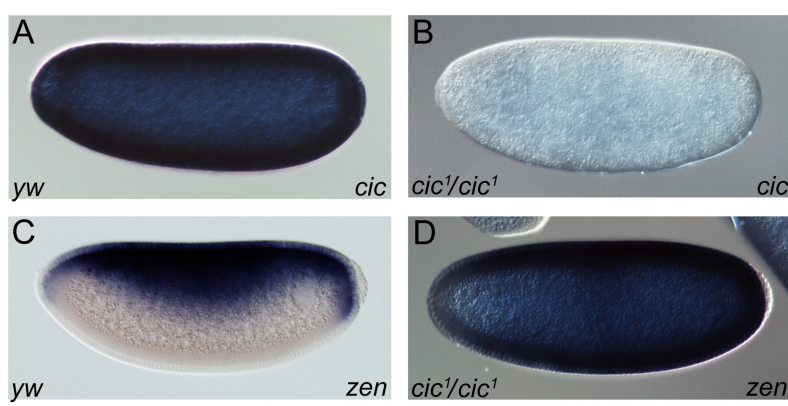

Fig. 3. Dl fails to repress zen expression in the lateral regions of cic mutant embryos. Whole-mount in situ hybridization was performed with wild-type (yw) and cic mutant embryos $\left(\mathrm{cic}^{1}\right)$. Wild-type (A) and homozygous cic mutant (B) embryos were hybridized with an anti-cic RNA probe. cic transcripts are ubiquitously found at high levels in early blastoderm embryos (A), while embryos homozygous for the cic mutant allele show no detectable cic mRNA (B). Wild-type (C) and homozygous cic mutant (D) embryos were hybridized with an anti-zen RNA probe. zen transcripts are dorsally distributed across approximately $40 \%$ of the wild-type syncytial blastoderm embryo's circumference (C). In homozygous $\mathrm{cic}^{1}$ mutant embryos, zen expression is derepressed, thereby expanding expression toward lateral and ventral regions of the embryos (D). These results suggest that cic expression is required for the repressive activity of the zen VRE. the $\mathrm{Cic}$ consensus sequence from the $\mathrm{B} 1 \mathrm{H}$ database will form a statistically significant cluster(s) with those of DI and Zld in the zen locus. A ClusterDraw analysis performed with $\mathrm{B} 1 \mathrm{H}$ PFMs of DI, Cic, and Zld (Fig. S3) over a $\sim 40-\mathrm{kb}$ genomic region encompassing the zen locus identifies two clusters, one of which corresponds to the previously identified VRE (Fig. 2A, dotted box). However, the P-value for the match probability of the cluster that corresponds to the VRE is high but does not seem to be statistically significant because of the low cluster P-value, suggesting that Cic may be dispensed to form clusters with $\mathrm{DI}$ and Zld. This possibility prompted us to test the ability, specifically, of PFMs of DI and Zld to form clusters in the identical genomic region. ClusterDraw analysis with PFMs of $\mathrm{Dl}$ and Zld resulted in comparable match probability values and cluster P-values (Fig. 2B) to those obtained from the former analysis with PFMs of DI, Cic, and Zld (Fig. 2A). These results suggest that the PFM of Cic produced by the $\mathrm{B} 1 \mathrm{H}$ system is functionally diverged from that of AT-rich sites at least in computational analyses because of a single nucleotide mismatch between the PFMs of Cic and AT-rich sites. Additional ClusterDraw analyses with PFMs of DI, AT sites (Fig. S2), and Zld present statistically significant values in both match probability and cluster formation (Fig. 2C), which is consistent with our previous prediction.

\section{Dl fails to repress zen expression in the lateral regions of cic mutant embryos}

Although computational analyses imply inability of Cic to form a cluster with $\mathrm{DI}$, it is apparent that the DNA sequence of each AT-rich site in the VRE has only one mismatch, compared with the $\mathrm{B} 1 \mathrm{H}$ consensus sequence of Cic. This fact prompted us to examine if $\mathrm{Cic}$ is genetically required for repression activity of the VRE. To this end, the zen expression pattern was examined in the context of wild-type and cic mutant embryos by whole-mount in situ hybridization with digoxigenin (DIG)-labeled RNA probes (Fig. 3). We found that cic mRNA is ubiquitously identified at high levels in early blastoderm embryos (Fig. 3A), consistent with maternal expression of the gene. At the identical stage, the embryo homozygous for the cic mutant allele $\left(\mathrm{Cic}^{1}\right)$ shows no detectable cic mRNA (Fig. 3B). zen is required for the development of dorsal tissues, including the amnioserosa and the optic lobe (13). During the syncytial blastoderm embryo stage, zen transcripts were distributed across approximately $40 \%$ of the embryo's circumference (Fig. 3C). In homozygous $\mathrm{CiC}^{1}$ mutant embryos, zen expression is de-repressed and, thus, expanded toward lateral and ventral regions of the embryos (Fig. 3D), which is indistinguishable from that observed in $\mathrm{dl}$ mutant embryos. These results suggest that cic is normally required to repress zen expression in the lateral regions of the embryos, thereby restricting zen expression to the dorsal ectoderm.

\section{Cic directly binds AT-rich sites in the zen VRE}

In Drosophila, two major Cic isoforms are expressed, Cic-S 
and Cic-L, which differ in their $\mathrm{N}$-terminal regions. The Cic isoforms share three highly conserved domains, called C1, C2, and a high mobility group (HMG) box (Fig. S4A). The $\mathrm{C} 1$ and C2 motifs have been known to be essential for transcriptional repression and MAP kinase recruitment, respectively (14). The HMG box, the third conserved domain, is involved in nuclear localization and DNA binding (15). To determine whether cic protein is able to directly bind the AT-rich sites in the zen VRE, electrophoretic mobility shift assay (EMSA) was performed with a recombinant Cic HMG-box region fused with glutathione S-transferase (GST) (Fig. S4A). Initially, a fusion protein containing GST and a Cic HMG-box region (amino acids 456-571) was overexpressed in E. coli and purified with glutathione-Sepharose resin (Fig. S4B and C). Approximately 40.4 $\mathrm{kDa}$ of purified Cic fusion protein was examined to bind a biotin-labeled DNA oligonucleotide containing either AT1, AT2, or AT3 sequences (refer to Fig. $1 \mathrm{~B}$ and $\mathrm{C}$ ) because previous studies showed that the lack of the three AT-rich sites abolished the repression activity of the VRE (7). The Cic HMG box fusion protein formed a DNA-protein complex with each of the three oligonucleotides (Fig. 4). The intensities of the
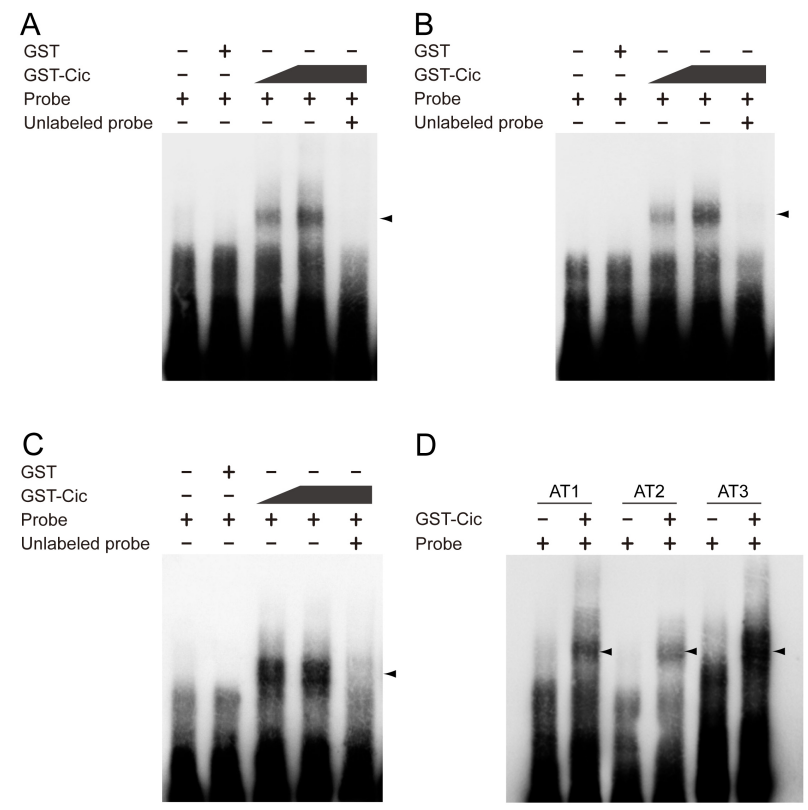

Fig. 4. Cic directly binds AT-rich sites in the zen VRE. Electrophoretic mobility shift assay (EMSA) was employed to test if $\mathrm{Cic}$ is able to directly interact with AT-rich sites in the VRE. EMSA was performed with purified GST-Cic fusion protein (Fig. S4) and biotin-labeled DNA fragments containing either AT1 (A), AT2 (B), or AT3 (C) sites (Table S1). Each DNA fragment was incubated with $0.2 \sim 0.4 \mu \mathrm{g}$ of GST-Cic protein. For competition experiments, 100-fold molar excess of unlabeled probes was added to the binding reactions. Binding affinities of GST-Cic fusion protein to each DNA fragment are compared in a single gel (D). Shifted DNA-protein complexes are indicated by arrowheads.
DNA-protein complexes seem to be proportionally augmented with an increasing amount of Cic fusion protein (Fig. 4A and B), except AT3 complexes (Fig. 4C). The addition of unlabeled probes to binding reaction mixtures abolished the formation of DNA-protein complexes (Fig. 4A-C). These results suggested that the Cic HMG box fusion protein is able to specifically and directly bind the three AT-rich sites found in the VRE.

The amount of DNA-protein complex formed by a small amount of Cic fusion protein $(0.2 \mu \mathrm{g})$ and the AT3 site is comparable to those of the complexes formed by large amounts of the fusion protein $(0.4 \mu \mathrm{g})$ and other AT sites. This observation raised the possibility that binding affinity of the AT3 site to the Cic fusion protein is higher than those of the AT1 and AT2 sites. To test this, complex formation of the Cic fusion protein with each of the three AT sites was compared simultaneously (Fig. 4D). The three AT-rich sites and Cic fusion protein formed three different amounts of DNA-protein complexes. AT3 and AT2 sites made the largest and least amount of DNA-protein complexes, respectively, and the amount of AT1-Cic protein complex was between AT3 and AT2. These results suggest that only a two or three nucleotide difference among the three sites (refer to Fig. 1C) may produce differential affinities for the Cic HMG box, thereby leading to different cooperative potentials with $\mathrm{DI}$ for repression of the VRE.

\section{DISCUSSION}

Although previous studies suggested that, like DI-mediated activation, DI-mediated repression also requires synergistic interactions between the $\mathrm{Dl}$ and so-called 'corepressor protein(s)' (7), the molecular identity of the corepressor protein remains unknown. Here, we present evidence that the Cic is involved in Dl-mediated repression.

Previous efforts to elucidate the corepressor protein that binds the zen VRE have identified several candidates. One putative corepressor protein is Dsp1 (Dorsal switch protein 1), which was identified in yeast two-hybrid screening (16). Dsp1 directly binds sites in the VRE and cooperates with DI or other NFKB subunits to repress transcription in cell culture assays. Dsp1 may be essential for optimal repression activity, but it does not seem to be necessary for VRE activity because minimal VRE sequences lacking DSP1 binding sites are still able to repress zen expression in the lateral regions of transgenic embryos (17). A second protein, NTF-1/Elf-1, was shown to bind to the ventral repression elements found in the zen and $d p p$ promoters. However, it is still unclear whether NTF-1/Elf- 1 is genetically required for VRE activity (18). Finally, it was recently reported that Dead ringer (Dri) and Cut (Ct) directly bind an AT2 site of the zen VRE (19). However, the VRE can still repress zen ventral expression in either dri or ct mutant embryos, suggesting that they may be not required for Dl-mediated repression in the context of the zen VRE. In this work, we showed that the VRE almost completely failed to repress zen ventral expression in cic mutant embryos and that 
Cic can directly bind three AT-rich sites in the VRE. These results strongly suggest that $\mathrm{Cic}$ is the corepressor that cooperates with Dl to repress zen ventral expression.

P-element-mediated germ-line transformation and reporter assays showed that three AT-rich sites, AT1, AT2, and AT3, are necessary and sufficient for repression of the VRE (7). Nucleotide sequence comparison between the three AT sites and $\mathrm{Cic}$ consensus sequence using the $\mathrm{B} 1 \mathrm{H}$ system indicates that there exists one nucleotide mismatch at the 3rd or 4th nucleotide position, relative to the $5^{\prime}$ end of each AT site (the $5^{\prime}$ end nucleotide is on the far left in each sequence; Fig. 1C and D). The $3 \mathrm{rd}$ and 4 th nucleotides of the $\mathrm{B} 1 \mathrm{H}$ consensus sequence of $\mathrm{Cic}(\mathrm{C}$ and $\mathrm{A}$ ) are nearly invariant, implying that the sequences of the three AT sites may not be a subset of the Cic consensus sequence. In fact, a ClusterDraw analysis with $\mathrm{B} 1 \mathrm{H}$ PFMs of $\mathrm{Dl}$, Cic, and Zld over a $40 \mathrm{~kb}$ genomic region encompassing a zen locus, failed to produce a statistically significant cluster p-value in the VRE (Fig. 2A). These results suggest that the binding affinities of the three AT sites to the cic proteins may be too low to be functional. Consistent with this, it has recently been proposed that high affinities of transcription factors are directly proportional to their functional relevance (20). How can the paradox be explained that the most critical AT sites for the repression activity of the VRE have low binding affinities to cic proteins? The simplest interpretation is that $\mathrm{Dl}$ is able to facilitate the binding of Cic to adjacent sites within the VRE, thereby leading to cooperative site occupancy of $\mathrm{DI}$ and Cic. In contrast to the low affinity AT sites, DI sites present in the VRE have high binding affinities (21). It is conceivable that, when DI binds the high affinity sites, it directly interacts with $\mathrm{Cic}$, inducing a conformational change in Cic to bind the low affinity AT sites more efficiently. Consistent with this interpretation, the insertion of a 5-bp spacer sequence between the AT2 and the closest DI binding sites disrupts the repression activity of an otherwise normal VRE (8). It is plausible to assume that the longer spacer sequence interferes with the cooperative interaction between $\mathrm{DI}$ and Cic.

EMSA performed with recombinant Cic showed that the AT2 site has the lowest affinity to Cic (Fig. 4D). However, previous mutagenesis analyses suggested that the AT2 site is particularly critical for VRE function, while AT1 and AT3 sites may be dispensable (8). If the interpretation described above is accurate, the fact that the low-affinity AT2 site is the most critical for repression could be explained by the cooperative site occupancy of $\mathrm{Dl}$ and Cic. An observation that the distance between the AT2 site and the nearby DI site is shorter than distances between other AT sites and their linked DI sites (7) strongly highlights the view that cooperative interaction between $\mathrm{Dl}$ and Cic can assist Cic to bind the lowest affinity AT2 site more efficiently.

\section{MATERIALS AND METHODS}

Detailed information is described in online Supplementary Material.

\section{ACKNOWLEDGEMENTS}

This work was supported by the National Research Foundation of Korea (NRF) Grants funded by the Korean Government (MSIP and MOE) (NRF-2010-0002792 and NRF-2012R1A1 A2038502).

\section{REFERENCS}

1. Ghosh, S., Gifford, A. M., Riviere, L. R., Tempst, P., Nolan, G. P. and Baltimore, D. (1990) Cloning of the p50 DNA binding subunit of NF-KB: homology to rel and dorsal. Cell 62, 1019-1029.

2. Rushlow, C. A., Han, K., Manley, J. L. and Levine, M. (1989) The graded distribution of the dorsal morphogen is initiated by selective nuclear transport in Drosophila. Cell 59, 1165-1177.

3. Hong, J. W., Hendrix, D., Papatsenko, D. and Levine, M. (2008) How the Dorsal gradient works: insights from postgenome technologies. Proc. Natl. Acad. Sci. U. S. A. 105 20072-20076.

4. Rushlow, C., Frasch, M., Doyle, H. and Levine, M. (1987) Maternal regulation of zerknüllt: a homoeobox gene controlling differentiation of dorsal tissues in Drosophila. Nature 330, 583-586.

5. St Johnston, R. D. and Gelbart, W. M. (1987) Decapentaplegic transcripts are localized along the dorsal-ventral axis of the Drosophila embryo. EMBO J. 6, 2785-2791.

6. Jiang, J., Rushlow, C. A., Zhou, Q., Small, S. and Levine, M. (1992) Individual dorsal morphogen binding sites mediate activation and repression in the Drosophila embryo. EMBO J. 11, 3147-3154.

7. Jiang, J., Cai, H., Zhou, Q. and Levine, M. (1993) Conversion of a dorsal-dependent silencer into an enhancer: evidence for dorsal corepressors. $E M B O$ J. 12, 3201-3209.

8. Cai, H. N., Arnosti, D. N. and Levine, M. (1996) Long-range repression in the Drosophila embryo. Proc. Natl. Acad. Sci. U. S. A. 93, 9309-9314.

9. Zhu, L. J., Christensen, R. G., Kazemian, M., Hull, C. J., Enuameh, M. S., Basciotta, M. D., Brasefield, J. A., Zhu, C., Asriyan, Y., Lapointe, D. S., Sinha, S., Wolfe, S. A. and Brodsky, M. H. (2011) FlyFactorSurvey: a database of Drosophila transcription factor binding specificities determined using the bacterial one-hybrid system. Nucleic Acids Res. 39, D111-117.

10. Liang, H. L., Nien, C. Y., Liu, H. Y., Metzstein, M. M., Kirov, N. and Rushlow, C. (2008) The zinc-finger protein Zelda is a key activator of the early zygotic genome in Drosophila. Nature 456, 400-403.

11. Jimenez, G., Guichet, A., Ephrussi, A. and Casanova, J. (2000) Relief of gene repression by torso RTK signaling: role of capicua in Drosophila terminal and dorsoventral patterning. Genes Dev. 14, 224-231.

12. Papatsenko, D. (2007) ClusterDraw web server: a tool to identify and visualize clusters of binding motifs for transcription factors. Bioinformatics 23, 1032-1034.

13. Wakimoto, B. T., Turner, F. R. and Kaufman, T. C. (1984) 
Defects in embryogenesis in mutants associated with the antennapedia gene complex of Drosophila melanogaster. Dev. Biol. 102, 147-172.

14. Astigarraga, S., Grossman, R., Diaz-Delfin, J., Caelles, C., Paroush, Z. and Jimenez, G. (2007) A MAPK docking site is critical for downregulation of Capicua by Torso and EGFR RTK signaling. EMBO J. 26, 668-677.

15. Stros, M., Launholt, D. and Grasser, K. D. (2007) The HMG-box: a versatile protein domain occurring in a wide variety of DNA-binding proteins. Cell Mol. Life Sci. 64, 2590-2606.

16. Lehming, N., Thanos, D., Brickman, J. M., Ma, J., Maniatis, T. and Ptashne, M. (1994) An HMG-like protein that can switch a transcriptional activator to a repressor. Nature 371, 175-179.

17. Jiang, J., Rushlow, C., Zhou, Q., Small, S. and Levine M. (1992) Individual dorsal morphogen binding sites mediate activation and repression in the Drosophila embryo. EMBO J. 11, 3147-3154.

18. Huang, J. D., Dubnicoff, T., Liaw, G. J., Bai, Y., Valentine, S. A., Shirokawa, J. M., Lengyel, J. A. and Courey, A. J. (1995) Binding sites for transcription factor NTF-1/Elf-1 contribute to the ventral repression of decapentaplegic. Genes Dev. 9, 3177-3189.

19. Valentine, S., Chen, G., Shandala, T., Fernandez, J., Mische, S., Saint, R. and Courey, A. (1998) Dorsal-mediated repression requires the formation of a multiprotein repression complex at the ventral silencer. Mol. Cell. Biol. 18, 6584-6594.

20. Fisher, W. W., Li, J. J., Hammonds, A. S., Brown, J. B. Pfeiffer, B. D., Weiszmann, R., MacArthur, S., Thomas, S., Stamatoyannopoulos, J. A., Eisen, M. B., Bickel, P. J., Biggin, M. D. and Celniker, S. E. (2012) DNA regions bound at low occupancy by transcription factors do not drive patterned reporter gene expression in Drosophila. Proc. Natl. Acad. Sci. U. S. A. 109, 21330-21335.

21. Ip, Y. T., Kraut, R., Levine, M. and Rushlow, C. A. (1991) The dorsal morphogen is a sequence-specific DNA-binding protein that interacts with a long-range repression element in Drosophila. Cell 64, 439-446.

22. Crooks, G. E., Hon, G., Chandonia, J. M. and Brenner, S. E. (2004) WebLogo: a sequence logo generator. Cenome Res. 14, 1188-1190. 